\title{
AFM Based Nano Telemanipulation for Indenting on the Human Chromosomes Using the Sliding Mode Impedance Controller
}

\author{
Samin Samadzade ${ }^{1}$, Alireza Habibnejad Korayem ${ }^{2}$, Moharam Habibnejad Korayem ${ }^{2, *}$ \\ ${ }^{1}$ Islamic Azad University -Science and Research Branch, Department of Mechatronics, Tehran, Iran \\ ${ }^{2}$ Robotic Research Laboratory, Center of Excellence in Experimental Solid Mechanics and Dynamics, School of Mechanical Engineering, \\ Iran University of Science and Technology, Tehran, 16846-13114, Iran
}

\begin{abstract}
In this article, the goal is to design an Atomic Force Microscopy (AFM) based nano telemanipulation system for indenting on the human chromosomes. The existence of un-modeled parameters in robot dynamics weakens the stability and performance of the system, therefore, by designing a sliding mode impedance controller on the slave side, the scaled down master position tracking, despite the presence of uncertainties is made possible, and with the design of an impedance controller on the master side, the scaled up reflected force of slave on operator is accomplished. The stability and transparency of the system are analyzed by extracting the teleoperation hybrid matrix. Specifically guaranteeing stability on the premise that some parts of the system are energy-producing is the main concern and treated by designing a suitable virtual coupling and haptic interface controller and by replacing the slave-side of the system with an appropriately trained neural network. Simulations performed in Matlab/Simulink verify the outcome of the paper.
\end{abstract}

Keywords Force Feedback, Impedance Controller, Virtual Environment, Neural Network, AFM, Nano Telemanipulation

\section{Introduction}

In the past few decades attention to nanomanipulation has enormously grown. It is well understood that interactions among objects in the nanocosm are quite different from those in the macrocosm. To be more specific, surface forces and intermolecular forces (Van der Waals force) present at the nano-scale dominate over gravitational and other more intuitive forces of the macro world and a human operator is not familiar with these novel nano force effects.

In this regard a large amount of research has been devoted to better understand the system and identify the obstacles and remedy them properly. Of the dominant challenges one is that there cannot be a visual feedback from the nano world. On the other hand the nano world produces too small positions and forces for the master side to be felt hence the introduction of position and force scaling factors which can instigate instability if not chosen appropriately. Furthermore nano-scale interactions involve complex nonlinear dynamics.

Nano-telemanipulation enables the operator to interact

* Corresponding author:

hkorayem@iust.ac.ir (Moharam Habibnejad Korayem)

Published online at http://journal.sapub.org/nn

Copyright $(2011$ Scientific \& Academic Publishing. All Rights Reserved with the environment indirectly and "feel" reaction forces as if he/she is actually present in the environment. The foremost issue in this respect is to build a suitable bridge between the nano and the macro world which can handle all the matters mentioned above and even more hence a compensation for inaccessibility of the nano environment. Force reflecting perception is important not only for enriching human operators to indirectly feel interaction between an AFM probe tip and samples at the nano scale, but also for reliable telemanipulation of fragile, soft and complex nano objects such as biological samples[1].

Nano telemanipulation systems allow interactions on much smaller scales than what is directly possible, in a way that the operator feels there is no intermediary between him and the environment[2]. The general configuration of a nano telemanipulation system is depicted in Figure 1. The most important goals in designing a nano telemanipulation system include: 1) stability of the closed-loop system irrespective of the behavior/dynamics of the operator and environment, 2) remote presence or transparency that gives a feeling of real presence of the operator in action.

With the invention of Scanning Tunneling Microscopy in 1990, the first master-slave nano telemanipulation system was presented by Hollis. Almost a decade after that, Sitti implemented his nano telemanipulation system with the help of Atomic Force Microscopy[3]. In his article, the cantilever 
had been assumed as a simple system of mass - spring and Damper. The inter-atomic forces between particle and probe tip were calculated based on the Lennard Jones potential and the DMT contact model.

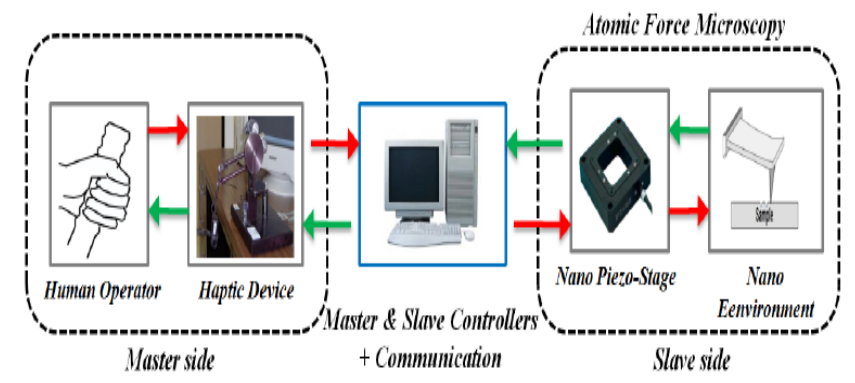

Figure 1. General configuration of nano telemanipulation system.

Since the existence of parametric uncertainty and un-modeled dynamics in the slave model will prevent proper slave position tracking, therefore, in the present article, an impedance controller based on the sliding mode for guaranteeing system robustness and for proper slave position tracking, and an impedance controller for proper force tracking on the master side have been considered.

The focus of this paper is to present a control configuration for an AFM-based telemanipulation system with a virtual slave-side (i.e. virtual slave and environment) considering the three main issues appropriately: stability, transparency and human perception. Our objective is that the human operator be able to fulfill the required task i.e. indentation of a surface with no trouble. For this he should be able to feel all the dominant forces present in nanoworld distinctively. We introduce a novel nano-telemanipulation configuration based on passivity concept and coupled stability theory. To simplify the result of his outstanding work it can be said the energy producing component must be connected to another component that is capable of dissipating the excess energy if stability is to be achieved. This implies that the haptic device and virtual coupling must combine to provide dissipation and this further implicates that they must satisfy strict passivity conditions.

\section{Controller Design}

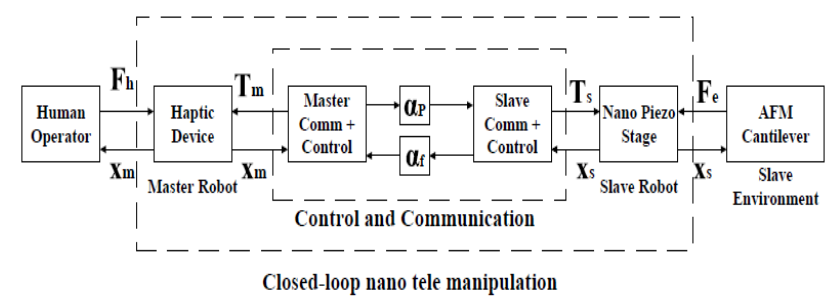

Figure 2. Closed-loop configuration of the nano telemanipulation system. $\underset{\text { tively }}{\alpha_{p}, \alpha_{f}}$ are the scale up/down coefficients of position and force, respec2.1. Configuration of the Nano Telemanipulation System
The general configuration of the nano telemanipulation system used in this article is according to Figure 2. The components of the system consist of: operator, master robot, master and slave controllers, piezoelectric stage (slave robot), AFM probe, and the sample as the environment. In this system, for proper interactions between the nano and macro environments it is necessary to scale up/down the variables of position and force exchanged between the two environments.

\subsection{Modeling of the Master and Slave Robots}

The dynamic model of the master robot is represented by (1), in which, $\mathrm{m}_{\mathrm{m}}$ is the effective mass of robot, $\mathrm{b}_{\mathrm{m}}$ is the damping coefficient, $\mathrm{F}_{\mathrm{m}}$ is the operator force, and $\mathrm{T}_{\mathrm{m}}$ is the control law.

$$
\mathrm{m}_{\mathrm{m}} \ddot{\mathrm{x}}_{\mathrm{m}}(\mathrm{t})+\mathrm{b}_{\mathrm{m}} \dot{\mathrm{x}}_{\mathrm{m}}(\mathrm{t})=\mathrm{T}_{\mathrm{m}}+\mathrm{F}_{\mathrm{h}}
$$

To calculate the inter-atomic forces, the cantilever dynamics of the atomic force microscope has been assumed as a system of mass-spring-damper according to (2) and Figure 3 , and the differential equation of the piezoelectric stage movement along the vertical is expressed as (3).

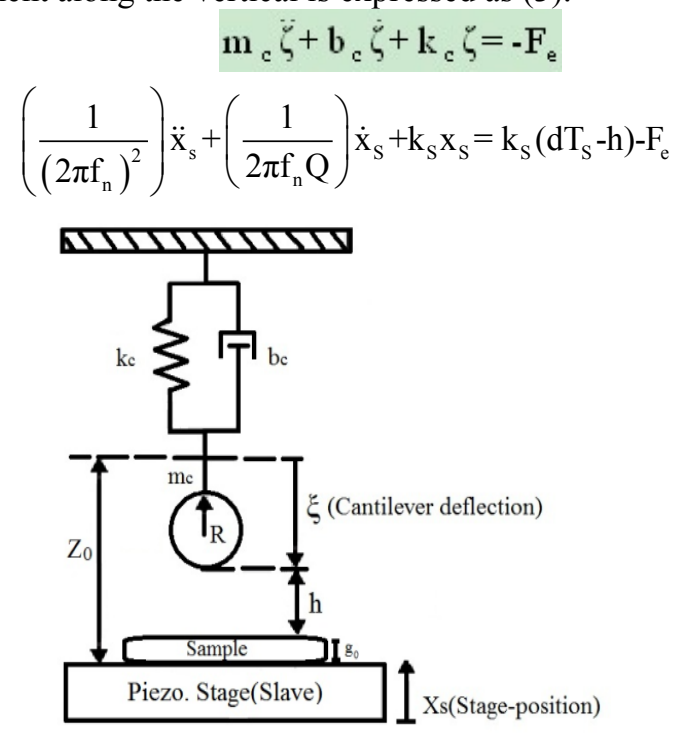

Figure 3. Mass-spring model for the AFM probe

$F_{e}, m_{c}, b_{c}, K_{c}, \xi, f_{n}, Q, x_{s}, T_{s}, d, h$ represent in the same order, the inter-atomic force between the probe tip and surface of sample, effective mass of cantilever tip, damping coefficient, spring constant, cantilever deflection, resonant frequency, quality factor, vertical displacement of piezo stage, control signal, displacement versus the input voltage and the hysteresis nonlinear term. The $\mathrm{m}$ and $\mathrm{s}$ indexes denote master and slave robot parameters, respectively[4-7]. Parameter $\mathrm{h}$ will be obtained from the Bouc-wen (4) after identifying the parameters $\alpha, \beta, \gamma$ are known.

$$
\dot{h}=\alpha d \dot{T}_{S}-\beta\left|\dot{T}_{S}\right| h-\gamma \dot{T}_{S}|h|
$$

When the probe tip approaches the surface, as long as the distance between the cantilever and surface remains in the $h>a_{0}$ range, the van der Waals and capillary non-contact 
forces act in attraction, and in the $h \leq \alpha_{0}$ range, because of the contact forces, the cantilever is in the contact region, and these forces were simulated based on the DMT model (5)

$$
F_{e}= \begin{cases}-\frac{H R}{6 h^{2}}-4 \pi R \gamma_{S L} & h>a_{0} \\ -\frac{H R}{6 a_{0}^{2}}+\frac{4 E^{*} \sqrt{R}}{3-3 v_{s}^{2}}\left(a_{0}-z-z_{c}\right)^{3 / 2} h & h \leq a_{0}\end{cases}
$$

$\mathrm{a}_{0}, \mathrm{E}, \mathrm{i}, \mathrm{h}, \mathrm{H}, \mathrm{R}, \gamma_{S L}$ are the intermolecular distance, the Young's modulus, Poisson's coefficient of the sample, the transient tip-sample separation, the Hamaker constant, the tip radius respectively and the surface energy between solid-liquid interface.

\subsection{Controller Design}

The desired impedance for the master robot is expressed as follows:

$$
\overline{\mathrm{m}}_{\mathrm{m}} \ddot{\mathrm{x}}_{\mathrm{m}}+\overline{\mathrm{b}}_{\mathrm{m}} \dot{\mathrm{x}}_{\mathrm{m}}+\overline{\mathrm{k}}_{\mathrm{m}} \mathrm{x}_{\mathrm{m}}=\mathrm{F}_{\mathrm{h}}-\alpha_{\mathrm{f}} \mathrm{F}_{\mathrm{e}}
$$

Where $\overline{\mathrm{m}}_{\mathrm{m}}, \overline{\mathrm{b}}_{\mathrm{m}}$, and $\overline{\mathrm{k}}_{\mathrm{m}}$ are the desired mass, damping coefficient, and stiffness of the dynamics of master robot, respectively. By subtracting (6) and (1) from each other, the control signal of the master robot is obtained as:

$$
\begin{aligned}
T_{m}(t)= & \left(b_{m}-\frac{m_{m}}{\bar{m}_{m}} \bar{b}_{m}\right) \dot{x}_{m}(t)+\left(\frac{m_{m}}{\bar{m}_{m}}-1\right) F_{h}(t) \\
& -\frac{m_{m}}{\bar{m}_{m}}\left\{\alpha_{f} F_{s}(t)+\bar{k}_{m} x_{m}(t)\right\}
\end{aligned}
$$

To obtain a proper slave position tracking, the desired impedance of the slave robot is expressed based on the position tracking error resulting from the interaction of the piezoelectric stage with the environment:

$$
\bar{m}_{s} \ddot{\tilde{x}}_{s}(t)+\bar{b}_{s} \dot{\tilde{x}}_{s}(t)+\bar{k}_{s} \tilde{x}_{s}(t)=-F_{e}(t)
$$

In the above relation, $\overline{\mathrm{m}}_{\mathrm{s}}, \overline{\mathrm{b}}_{\mathrm{S}}, \overline{\mathrm{k}}_{\mathrm{s}}$, and $\tilde{\mathrm{x}}_{\mathrm{S}}:=\mathrm{x}_{S}-\alpha_{\mathrm{p}} \mathrm{x}_{\mathrm{m}}$ are the desired mass, damping coefficient, stiffness, and the positioning error of the slave robot dynamics, respectively. By inserting relation (8) in relation (3), the equivalent control law is obtained. However, since the exact values of slave parameters are not available, they have been estimated. The sliding mode controller should be designed in such a way that the desired impedance model is achieved over the sliding surface[5]; for this purpose, the sliding surface has been defined as:

$$
\begin{aligned}
& s(t):=\frac{1}{\bar{m}_{s}} \int_{0}^{t} I_{e}(\tau) d \tau=\dot{\tilde{x}}(t)+\frac{\overline{\mathrm{b}}_{s}}{\overline{\mathrm{m}}_{s}} \tilde{\mathrm{x}}(\mathrm{t}) \\
& +\int_{0}^{\mathrm{t}}\left[\overline{\mathrm{k}}_{\mathrm{s}} \tilde{\mathrm{x}}(\mathrm{t})+F_{e}(\tau)\right] \mathrm{d} \tau
\end{aligned}
$$

If the sliding condition $\dot{\mathrm{s} s} \leq-\eta|\mathrm{s}|$ is satisfied, phase trajectories will incline towards the sliding surface $\mathrm{s}$. In this relation, the value of $\eta$ is constant. To make sure of a control input that will maintain the sliding condition despite system uncertainties, the discontinuous term $-K_{g} \operatorname{sgn}(s)$ is added to the equivalent control law. By considering all the above cases, the slave control law will be obtained as:

$$
\begin{aligned}
T_{s}= & \frac{1}{\hat{k}_{S} d}\left\{\frac{\alpha_{p} \hat{m}_{S}}{\bar{m}_{m}}\left\{-\bar{b}_{m} \dot{X}_{m}-\bar{k}_{m} X_{m}+F_{h}-\alpha_{f} F_{e}\right\}-\right. \\
& \frac{\hat{m}_{S}}{\bar{m}_{s}}\left\{\bar{b}_{s} \dot{\tilde{X}}+\bar{k}_{s} \tilde{X}+F_{e}\right\}+b_{s} X_{s}+k_{s} X_{s}+F_{s}+k_{s} h_{B W} \\
& -k_{g} \operatorname{sat}\left(\frac{s(t)}{\varphi}\right)
\end{aligned}
$$

In the above relation, $\mathrm{k}_{\mathrm{g}}$ and $\varphi$ represent the nonlinear gain and boundary layer thickness, respectively.

\section{Analysis of stability and Transparency}

\subsection{Llewellyn Absolute Stability Criteria}

The Llewellyn criteria expresses as the following[5], the necessary and sufficient condition for absolute stability of the teleoperator based on the hybrid matrix components $h_{i i}$ :
a) $R\left[h_{11}\right] \geq 0$
b) $R\left[h_{22}\right] \geq 0$
c) $f(w)=2 R\left[h_{11}\right] R\left[h_{22}\right]-R\left[h_{12} h_{21}\right]-\left|h_{12} h_{21}\right| \geq 0$

For the stated (our) system, the hybrid matrix components are obtained as follows:

$$
\begin{array}{ll}
h_{11}=\bar{m}_{m} s+\bar{b}_{m}+\frac{\bar{k}_{m}}{s}, & h_{21}=-\alpha_{f} \\
h_{21}=\alpha_{p}, & h_{22}=\frac{s}{\bar{m}_{S} s^{2}+\bar{b}_{S} s+\bar{k}_{s}}
\end{array}
$$

The above relations show that by selecting positive parameters for desired impedances of the master and slave robots, the designed controller will be stable for every passive user and environment, and for all the scale up/down coefficient values. It can be easily proven that by selecting a large enough $\mathrm{K}_{\mathrm{g}}$ value, the system stability is assured.

\subsection{Analysis Transparency}

Quantitatively, transparency can be expressed as conformity between the environment impedance and the impedance which is transferred to the operator[6]. Therefore, considering Figure 2 and the hybrid matrix in the two cases of non-contact and contact, the system performance is evaluated.

$$
\begin{aligned}
\left.Z_{\text {to } \min }\right|_{Z_{e}=0} & =h_{11} \\
\left.Z_{\text {towidth }}\right|_{Z_{e} \rightarrow \infty} & =\frac{-h_{12} h_{21}}{h_{22}}
\end{aligned}
$$

With this explanation, a satisfactory performance is accomplished when

$$
\left|Z_{\text {to min }}\right| \rightarrow 0,\left|Z_{\text {towidth }}\right| \rightarrow \infty
$$

\section{Simulation Results}

In this section, the simulation of the presented nano telemanipulation system is carried out. The human operator is modeled as a damper-spring system, with a hardness coeffi- 
cient of $50 \mathrm{~N} / \mathrm{m}$ and damping coefficient of $75 \mathrm{~N} / \mathrm{m}[8]$. The desired impedance parameters to achieve stability and transparency in the designed controllers are as follows:

$$
\begin{gathered}
h=150 e^{-9}-(x s+\xi), H=1 e^{-21}, R=10 e^{-9}, f_{n}=450 \mathrm{~Hz}, \\
Q=20, m_{c}=1.2 e^{-7}, b_{c}=1.7 e^{-5}, k_{c}=40, \bar{m}_{s}=1 e^{-4}[\mathrm{~kg}] \\
\bar{b}_{s}=1 e^{-4}\left[\frac{N s}{m}\right], \bar{k}_{s}=6 e^{2}\left[\frac{N}{m}\right], m_{m}=0.5[\mathrm{~kg}], b_{m}=0.2\left[\frac{N s}{m}\right] \\
\bar{m}_{m}=0.02[\mathrm{~kg}], \bar{b}_{m}=0.05\left[\frac{N s}{m}\right], \bar{k}_{m}=0.04\left[\frac{N}{m}\right], \\
\alpha_{\mathrm{p}}=1 \mathrm{e}^{-6}, \alpha_{\mathrm{f}}=1 \mathrm{e}^{7}, \varphi=0.1, \mathrm{~kg}=250
\end{gathered}
$$

Simulations are performed on two different human chromosomes with Young modulus[9] $\mathrm{E}=13$ ? $0^{5}$, $\mathrm{E}=4$ ? $0^{5}$, and $v=0.4$ for underestimated parameters $\hat{\mathrm{m}}_{\mathrm{s}}=0.5_{\mathrm{s}}, \hat{\mathrm{b}}_{\mathrm{s}}=0.5_{\mathrm{s}} \& \hat{\mathrm{k}}_{\mathrm{s}}=0.5_{\mathrm{s}}$. The operator moves the master robot from position (0) to a position of $15 \mathrm{~cm}$ within a time span of zero (0) to 5 seconds and in the time span of $5 \mathrm{~s}$ to $6.5 \mathrm{~s}$, he senses the Reflected force resulting from the slave robot making contact with the environment, and finally, in the time span of $6.5 \mathrm{~s}$ to $11.5 \mathrm{~s}$, the operator returns the master robot to position zero $(0)$.

In Figure 4, the master and slave robot position tracking, and in Figure 5, the master and slave robot force tracking with present uncertainty have been reviewed. Figures 6 and 7 demonstrate that regardless of slave parametric uncertainty and hysteresis, the position and force tracking is acceptable.

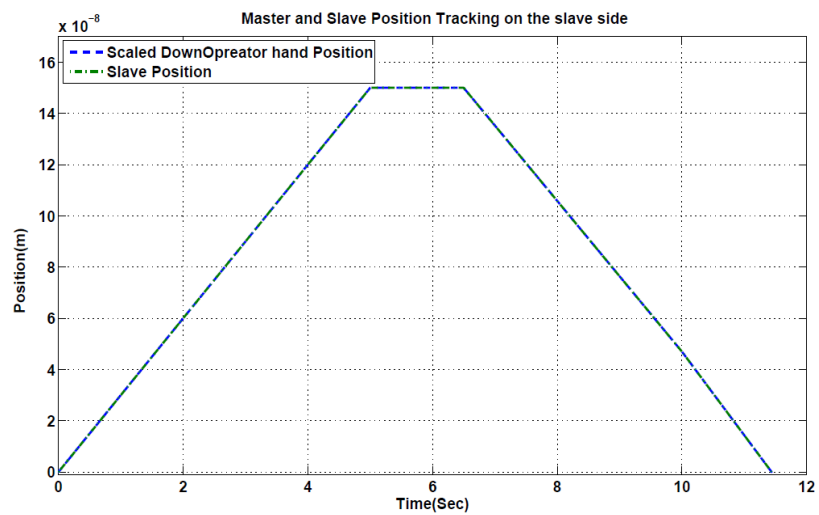

Figure 4. Slave position tracking in the environment $E=13 e^{5}$

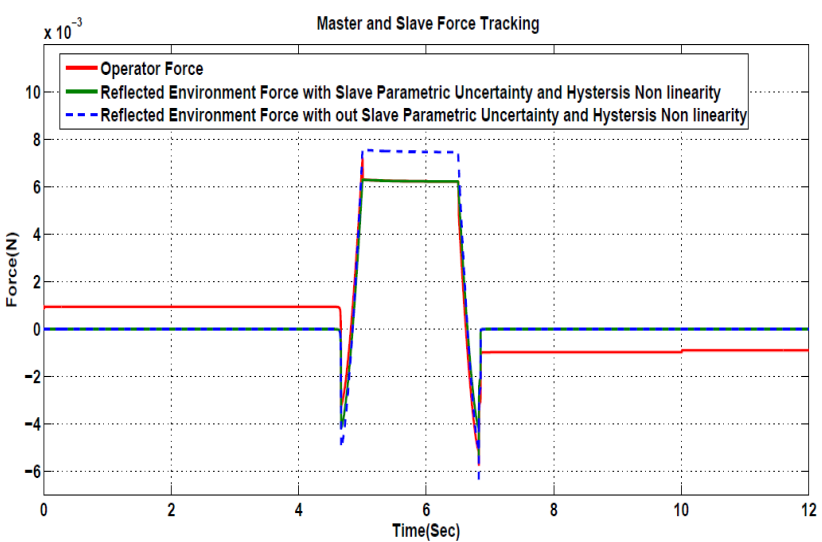

Figure 5. Master force tracking and evaluation of hysteresis nonlinearity

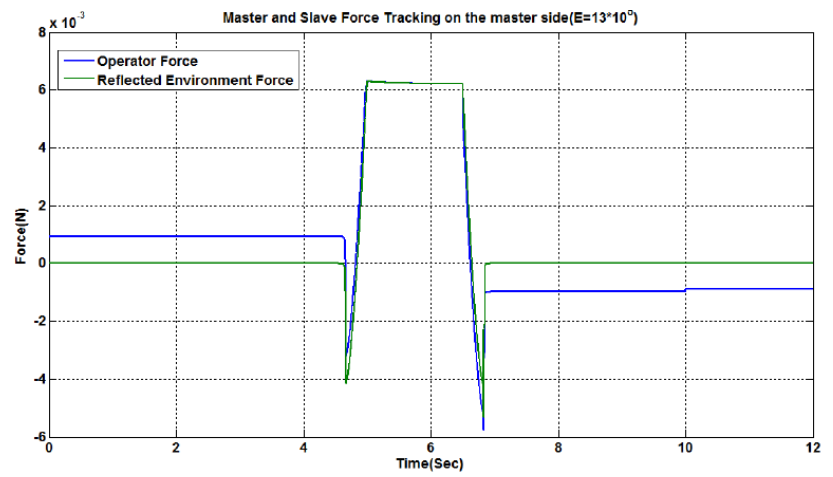

Figure 6. Master force tracking in the presence of hysteresis nonlinearity in the $E=13 e^{5}$ environment

In Figures 6 and 7, the effects of contact and non-contact forces on the cantilever approach in two different human chromosomes can be observed.

Figure 8 represents both methods to derive velocity. This figure shows velocity is approximated smoothly with small deviations from differentiated position.

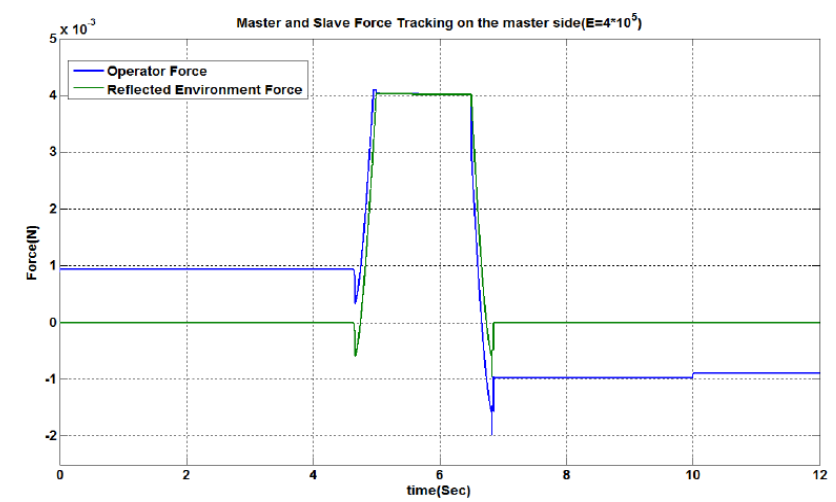

Figure 7. Master force tracking in the presence of hysteresis nonlinearity in the $E=4 e^{5}$ environment nonlinearity

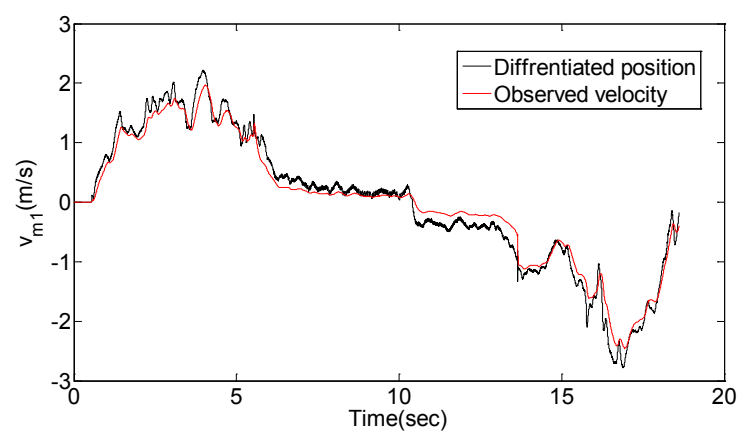

Figure 8. velocity obtained from two methods (differentiating and observing)

To produce acceptable outputs, it is required that the neural network be trained aptly. Therefore we have to supply the neural network with more as well as richer data. In that regard the original nano-telemanipulation system was tested by an operator. In every experiment indentation depth or operation speed (slave speed) was changed so that variegated inputs would be provided. 41 experiments were run all in all. The fixed parameter in these experiments was 
chosen as follows:

$$
\begin{aligned}
& m_{c}=4.5 \times 10^{-11}, b_{c}=1.4 \times 10^{3}, k_{c}=40, f_{p}=450, Q=20, k_{p}=1, \\
& H=10^{-19}, R=20 \times 10^{-9}, a_{0}=1 A^{0}, v=0.2, g_{0}=10 \mathrm{~nm}, \\
& k_{\text {max }}=200, k_{\min }=40, k_{e}^{\min }=2 \times 10^{-3}, k_{e}^{\max }=2 \times 10^{3}, \\
& K=150, \alpha_{p}=0.6 \times 10^{-6}, \alpha_{f}=6 \times 10^{6}
\end{aligned}
$$

Since $k_{s}$ varies with variation of indentation depth, it is approximated as its value when the penetration depth is 2.5 $\mathrm{nm}$. The augmented virtual environment and the virtual coupling updating rate is $1000 \mathrm{~Hz}$.

Figures 9-12 depict the results of an experiment on a virtual sample. In Figure 9 master and slave positions are shown. Position drift is spotted when the slave is in contact with the environment where free motion is performed with perfect coordination.

Figure 10 illustrates the tip-surface distance in the same experiment. In this graph negative values denote penetration depth in the sample. The maximum indentation depth is nearly $2.7 \mathrm{~nm}$.

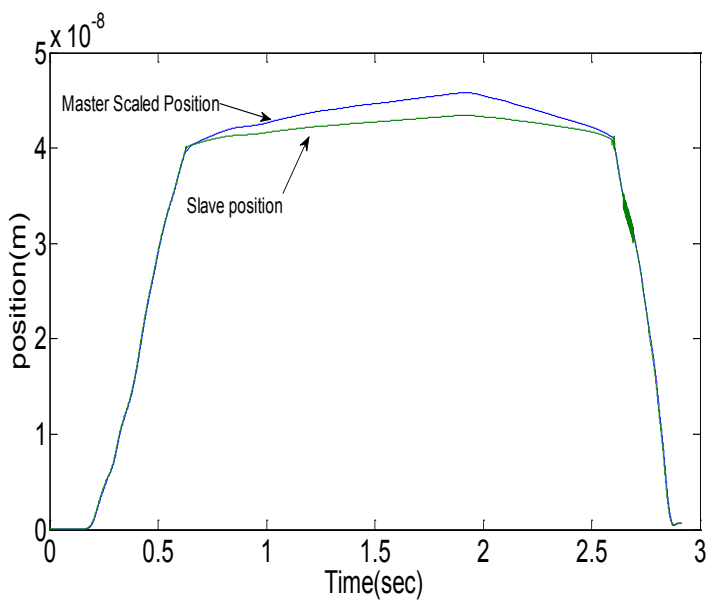

Figure 9. Master and slave position in an indentation task on a virtual sample with $\mathrm{E}=10 \mathrm{GPa}, \mathrm{v}=0.2$

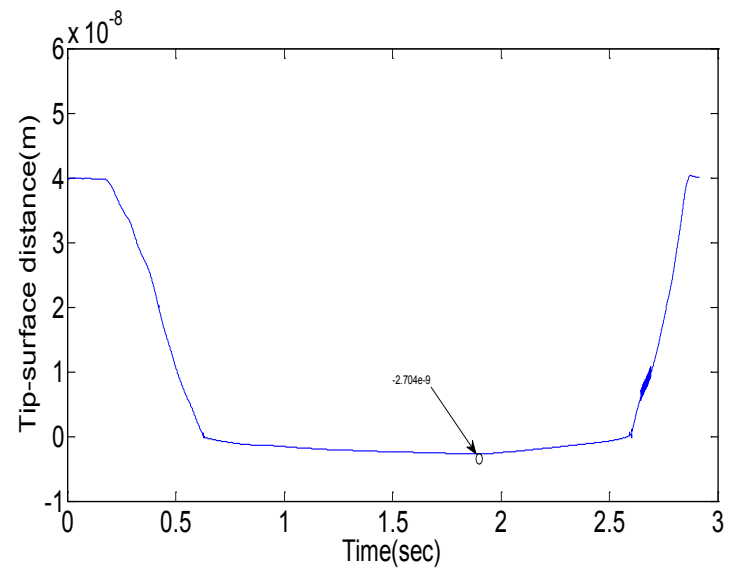

Figure 10. Tip-surface distance in an indentation task on a virtual sample with $\mathrm{E}=10 \mathrm{GPa}, \mathrm{v}=0.2$

Figure 11 shows the reflected force to the operator. The initial small positive peak (within $0.62-0.65$ ) is attributed to the Van der Waals force, the next large negative peak (within
$0.65-2.57)$ is pertaining to the contact force and the last small negative peak (within 2.57-2.62) is pertinent to the adhesion force.

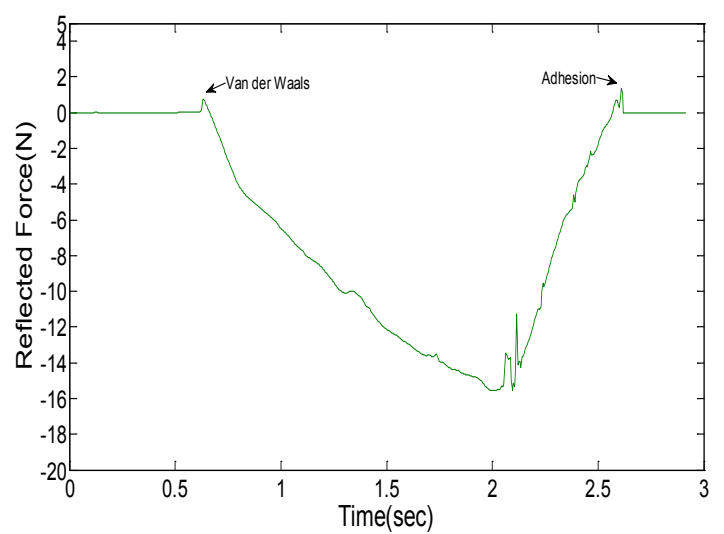

Figure 11. Reflected force to the operator in an indentation task on a virtual sample with $\mathrm{E}=10 \mathrm{GPa}, \mathrm{v}=0.2$

Finally in Figure 12 adaptive damping coefficients implemented on the master to stabilize the system in the experiment are plotted. Zero values imply the system's own dissipation including inherent master and human damping is sufficient to stabilize the system. In other cases necessary damping is applied and the system always remains stable.

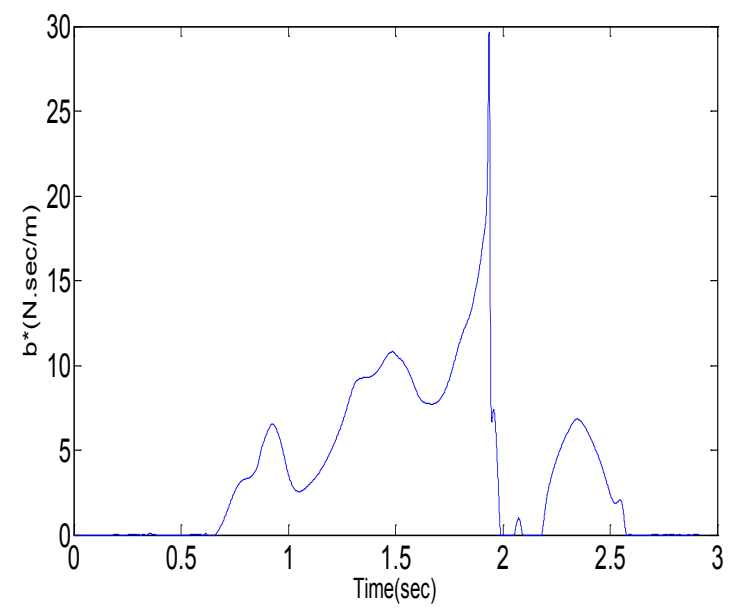

Figure 12. Adaptive damping implemented on the master manipulator in an indentation task on a virtual sample with $\mathrm{E}=10 \mathrm{GPa}, \mathrm{v}=0.2$

\section{Conclusions}

A novel approach for designing a nano-telemanipulation system is proposed which can guarantee a stable interaction with nonlinear and inherently active nano virtual environments. The method relies only on the haptic interface damping (either inherent or through a damping injection controller) to stabilize the nano-telemanipulation system.

In this article, a nano telemanipulation system was studied with the consideration of slave parametric uncertainty and un-modeled dynamics. The system stabilities were separately evaluated in relation to the Llewellyn criteria and transparency. Performed simulations demonstrated the sta- 
bility and proper functioning of the system despite the uncertainties.

\section{REFERENCES}

[1] S. G. Kim, and M Sitti, "Task-based and stable telenanomanipulation in a nanoscale virtual environment," IEEE Transactions on Automation Science and Engineering, vol. 3, no. 3, pp. 240-247, 2006.

[2] D. Cagdas Onal, and S. Metin, “A Scaled Bilateral Control System for Experimental One-dimensional Teleoperated Nanomanipulation," International Journal of Robotics Research vol. 28, no. 4, pp. 484-497, April 2009.

[3] M. Sitti, S. Horiguchi, and H. Hashimoto, "Nano telemanipulation using virtual reality interface," IEEE International Symposium on Industrial Electronics, (ISIE 98), vol.1, pp. 171-176, Jul 1998 .
[4] Y. Song and B. Bhushan, "Atomic force microscopy dynamic modes: modeling and applications," Journal of Physics: Condensed Matter, vol. 20 no.22, 5012, June 2008.

[5] H. Chul Cho and J. Hyeon Park, "stable bilateral teleoperation under a time delay using a robust impedance control," Mechatronices, vol.15, no.5, pp. 611-625, June 2005.

[6] J.J.E. Slotine, and Li W., “Applied nonlinear control," NJ: Prentice-Hall; 1991.

[7] K. Hashtrudi_Zaad, and S.E. Salcudean, "Analysis and Evaluation of Stability and Performance Roubustness for Teleoperation Control Architectures," Proceedings of IEEE International Conference on Robotics and Automation, pp. 3107-3113, May 2000, San Francisco.

[8] D. Lee, and W. Spong, "Passive Bilateral Teleoperation with Constant Time Delay," IEEE Transactions on Robotics. vol. 22, no. 2, pp. 269-281, April 2006.

[9] A. 1kai, X. Ming xu, and K. Mitsui, "Measurements of mechanical parameters of biological structures with atomic force microscope," Scanning Microscopy, vol. 12, no.4, pp. 585-598, 1998. 